\title{
Burkitt lymphoma of the thyroid (a rapidly growing painless thyroid mass during pregnancy)
}

Bellarbi $\mathrm{D}^{1}$, Si youcef $\mathrm{H}^{1}$, Azzoug $\mathrm{S}^{1}$, Chentli $\mathrm{F}^{1}$, Amalou $\mathrm{M}^{2}$, Merad $\mathrm{F}^{2}$

1 Endocrine and Metabolic diseases department Bab El Oued Hospital Algiers Algeria

2 Department of general surgery Bab El Oued Hospital Algiers Algeria

\section{INTRODUCTION}

Primary thyroid lymphomas (PTL) are rare tumors, accounting for $2.5 \%$ of all non-Hodgkin's lymphomas and fewer than $2 \%$ of all malignant thyroid tumors. Primary thyroid's Burkitt lymphoma represents a very unusual type of PTL. Only very few cases of primary thyroid's Burkitt lymphoma have been reported during pregnancy.

\section{OBSERVATION}

We present a 30 years old female patient who developed a rapidly enlarging painless neck mass. This mass was noticed by the patient few days after a severe flue at $20^{\text {th }}$ week of pregnancy. We received our patient at 29th week and 04 days of her first pregnancy. She had no other diseases or family history of thyroid disease. Physical examination revealed an enlarged thyroid gland (Fig 1). Compressive signs were limited to respiratory distress. Ultrasound of the neck showed increased dimensions of thyroid gland. Her thyroid function tests were normal. Anti-thyroid peroxidase antibodies were positive. Fine needle aspiration biopsy within the thyroid gland was compatible with thyroiditis. Compression was objected on MRI cervical examination which revealed a voluminous goiter with discreet lamination of the trachea (Fig 2). So she had a prophylactic ceasarean delivery of a healthy, premature male infant at 32 weeks of gestation. Urgent thyroidectomy (Fig 3 ) was performed and the diagnosis of thyroid's Burkitt lymphoma was made on pathology. Subsequent staging procedures included CT imaging of the neck, chest and abdomen which revealed nodes enlargement in the chest. Chemotherapy was initiated six weeks after surgery.
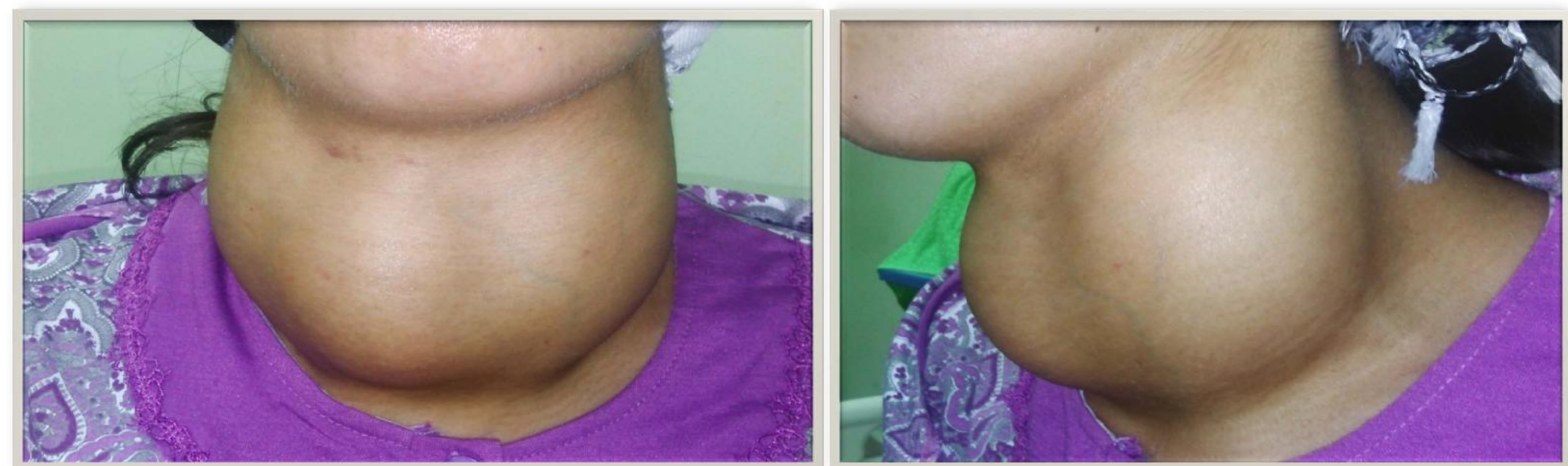

Fig 1

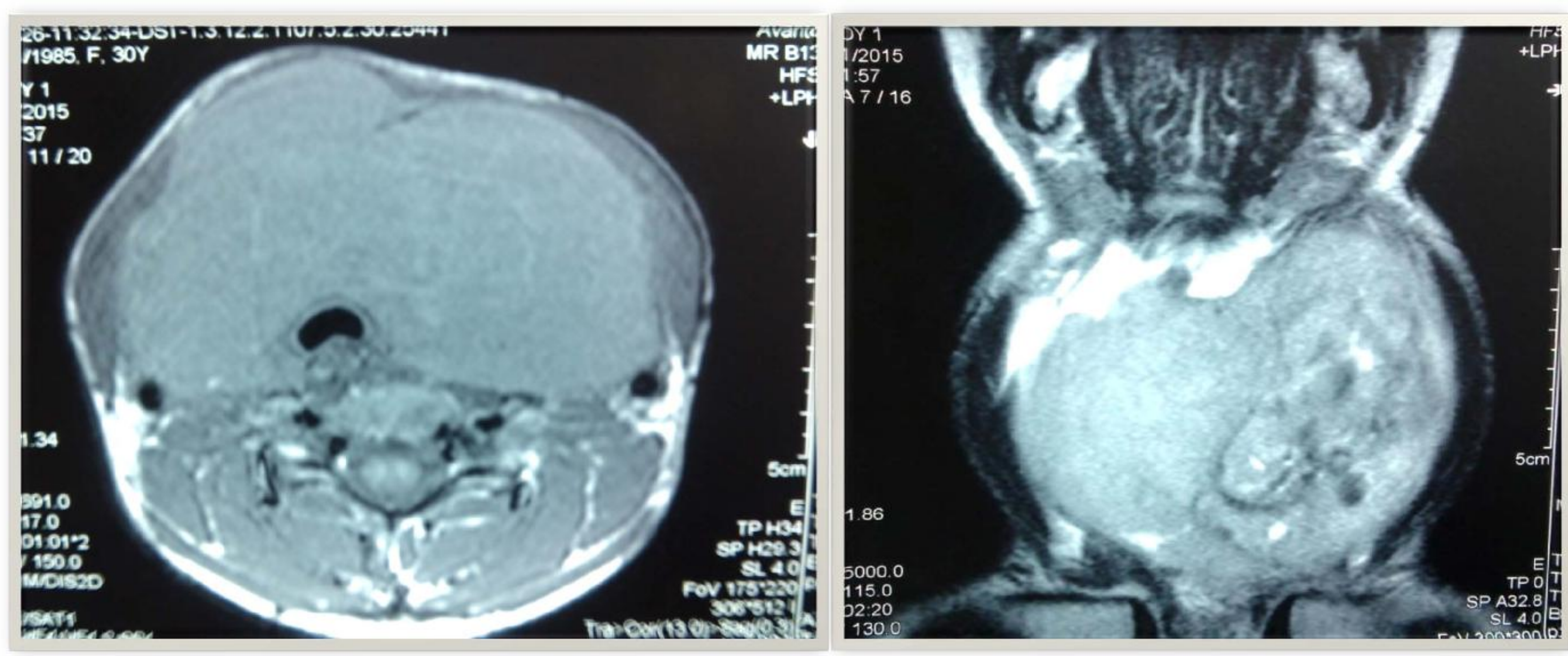

Fig 2

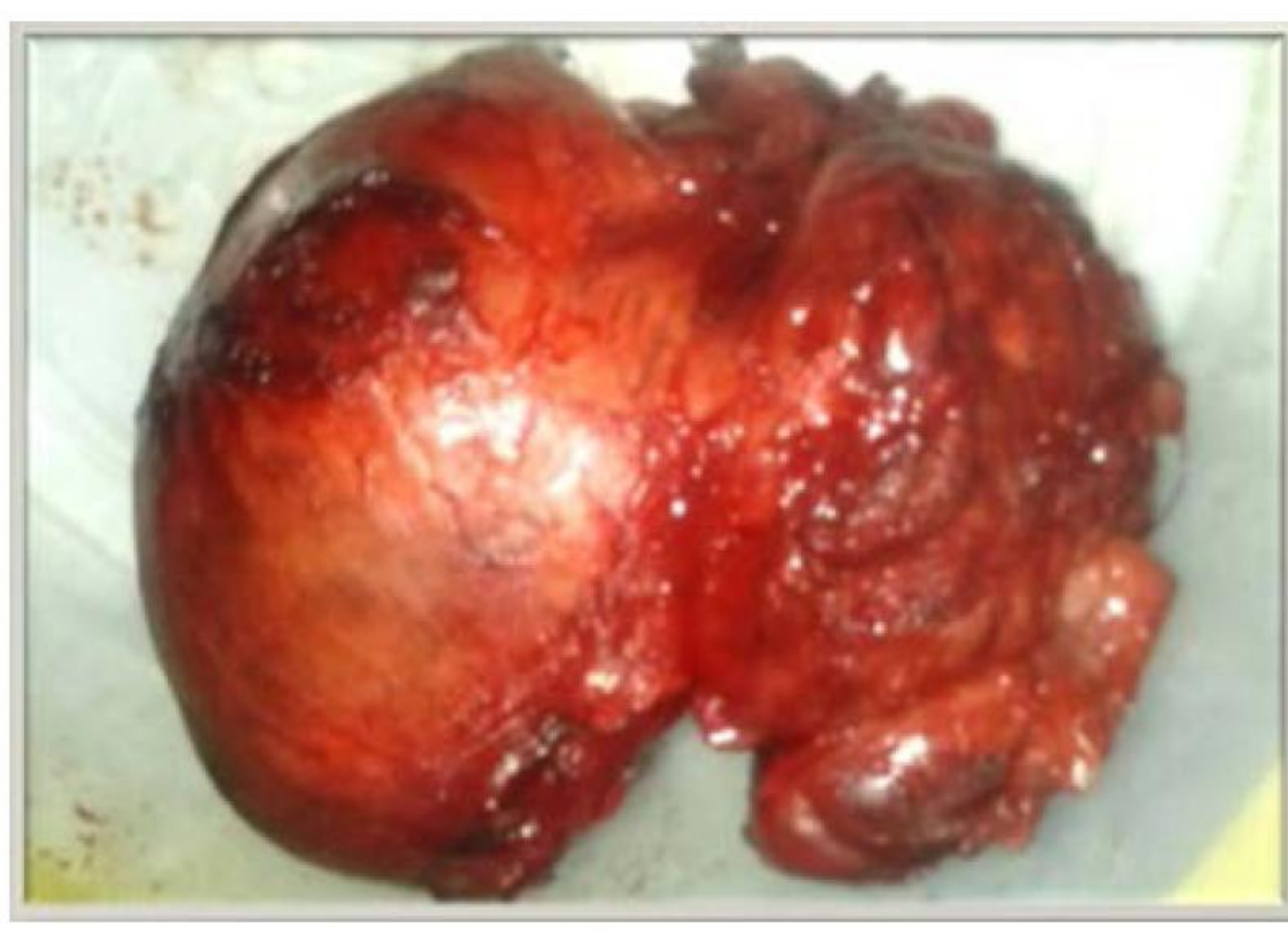

Fig 3

CONCLUSION

Thyroid's Burkitt lymphoma is rare; its occurrence during pregnancy is even rarer. Its diagnosis and management is difficult. A multidisciplinary team is tremendously important in order to take the right decisions to save both the baby and the mother. 